\title{
Duodenal gangliocytic paraganglioma with lymph node metastasis: A systematic case review
}

\author{
Luke Hartford, Alexsi Sherazadishvili, Ken Leslie
}

\begin{abstract}
Introduction: Duodenal gangliocytic paragangliomas (DGPs) are rare tumors, most commonly located in the 2nd portion of the duodenum. Their origin is poorly understood and management is uncertain. Typically benign, they infrequently metastasize to lymph node and distant sites. Objective: A systematic literature search for DGPs with lymph node metastases was performed. Epidemiological, diagnostic, management, surveillance and outcome data were recorded. The histopathology and immunohistochemistry of these tumors and possible predictors of lymph node metastases were revisited. Thirty three cases of DGPs with lymph node metastases were included. Mean patient age was 48 years, with no predilection for sex. Discussion: Presenting complaints included abdominal discomfort and gastrointestinal bleeding/anemia. Tumor size (maximum diameter) ranged from 1 to $9 \mathrm{~cm}$, with a mean
\end{abstract}

Luke Hartford ${ }^{1}$, Alexsi Sherazadishvili ${ }^{2}$, Ken Leslie ${ }^{3}$

Affiliations: ${ }^{1}$ General Surgery Resident, Department of General Surgery, London Health Sciences Centre, 800 Commissioners Road East, Room E2-213, Zone E, London, Ontario, Canada; ${ }^{2}$ MD Candidate, Class of 2019, Schulich School of Medicine and Dentistry, University of Western Ontario, 1151 Richmond St. London, Ontario, Canada; ${ }^{3}$ Chair/ Chief Division of General Surgery, Associate Professor, London Health Sciences Centre, 800 Commissioners Road East, Room E2-213, Zone E, London, Ontario, Canada.

Corresponding Author: Luke Hartford, General Surgery Resident, Department of General Surgery, London Health Sciences Centre, 800 Commissioners Road East, Room E2-213, Zone E, London, Ontario, Canada, N6A 5A5, Email: luke.hartford@Ihsc.on.ca

Received: 14 October 2017

Accepted: 25 November 2017

Published: 21 December 2017 of $3.1 \mathrm{~cm}$. Serum/urine tumor markers and hormones were inconsistent. However, there was evidence of neuroendocrine activity. Esophagogastroduodenoscopy successfully identified the lesion in $23 / 23$ reported cases, but had no role in tissue diagnosis. Computed tomography scan and endoscopic ultrasound correctly identified a mass in $84 \%$ and $100 \%$ of reported cases and were successful in detecting lymph node involvement in $47 \%$ and $64 \%$ of cases. Pancreatoduodenectomy was the definitive treatment in $87 \%$ of the cases. Possible predictors of lymph node metastasis included tumor extension and angiolymphatic invasion, as well as changes noted in immunohistochemistry. Conclusion: We suggest diagnostic aids, management and surveillance for DGPs with lymph node metastases. Due to uncertain malignant potential, surgical management and treatment adjuncts like chemotherapy and radiation should be investigated. Surveillance has not been well established and should be focused on diagnostic imaging and close clinical follow-up.

Keywords: Duodenal, Gangliocytic, Malignancy, Paraganglioma

\section{How to cite this article}

Hartford L, Sherazadishvili A, Leslie K. Duodenal gangliocytic paraganglioma with lymph node metastasis: A systematic case review. Int $\mathrm{J}$ Hepatobiliary Pancreat Dis 2017;7:53-61.

Article ID: 100074IJHPLHO2017

doi:10.5348/ijhpd-2017-74-RA-10 


\section{INTRODUCTION}

Gangliocytic paragangliomas are rare tumors, first described by Dahl et al., and are most commonly located in the 2nd portion of the duodenum $[1,2]$. While their origin is poorly understood, they are composed of epithelioid, spindle shaped and ganglion-like cells of various proportions $[2,3-7]$. Typically exhibiting benign behaviour, and treated with simple excision, these tumors uncommonly metastasize to lymph node, and there have been reports of recurrence and metastasis to distant organs, with one report of mortality $[1-3,8,9]$. With approximately 200 case reports of duodenal gangliocytic paragangliomas (DGPs), and $5-7 \%$ containing lymph node metastasis, the majority of medical literature currently surrounds pathological characteristics [10]. Limited information is available to guide the clinical and surgical management of these patients, and the significance of lymph node metastases and long-term outcomes is largely unknown. The presence of lymph node metastasis is consequential, as currently, the majority of these patients are treated with a pancreaticoduodenectomy, instead of local excision. Herein, we summarize the literature in a systematic manner, providing a review on evidence surrounding epidemiology, diagnosis, management, surveillance and extended outcomes. We also revisit the histopathology and immunohistochemistry of these tumors, and the significance of angiolymphatic invasion, mitotic rate, nuclear pleomorphism, tumor size and depth of invasion as a predictor or lymph node involvement.

\section{METHODS}

\section{Literature search strategy and selection}

A systematic literary research was performed independently by two different investigators in Medline, EMBASE, Web of Science Database and Google Scholar with no language restrictions. The search terms included the following: 'gangliocytic', 'paraganglioma', 'lymph node', 'paraganglioneuroma', 'pheochromocytoma', 'duodenum', 'pancreatic', 'ampulla of Vater', 'duodenal papilla', 'sphincter', 'Oddi', 'neoplasm', 'cancer', 'adenocarcinoma', 'sarcoma', 'tumor', 'metastasis', 'malignant', combined with and/or. Search restrictions included all species other than humans, with no restrictions on article type. The references of all selected articles were reviewed. Duplicate studies were eliminated. Agreement between the two reviewers was measured with the Cohen's kappa coefficient. All retrieved articles were selected if they met inclusion criteria.

\section{Selection criteria}

Selected studies contained patients with DGPs, with lymph node metastasis, both confirmed by histopathology. All abstracts/conference proceedings, case reports, reviews and meta-analyses of all languages were included, if they contained management and outcome data. No selection on age, sex or ethnicity was adopted.

\section{Data extraction}

Year of publication and study type were reported. Study data included patient characteristics such as age, sex, and symptoms. Diagnostic imaging, biopsies, operation, postoperative course, radiation/chemotherapy and local recurrence were reported. After research ethics board approval, authors were contacted on three separate occasions and the most current disease free time interval was included. Gross pathology, histopathology and immunohistochemistry findings were summarized. Levels of evidence and grades of recommendations were in keeping with the Oxford Centre for Evidence-based Medicine guidelines [11].

\section{Statistical analyses}

Descriptive patient and tumor characteristics were reported. These were compared to those cases with lymph node metastases from Okubo et al. [10]. Outcomes were reported in months, using both clinical symptoms and diagnostic imaging, as a method of surveillance.

\section{RESULTS}

\section{Selection}

Searching key terms in Medline, EMBASE and Web of Science databases yielded 2,474 articles. After duplicate studies were removed 1,276 remained. After screening titles and abstracts, 34 articles were selected as potentially eligible for the study, and 29 reports met inclusion criteria on further review. Two additional articles were identified after reviewing references. An additional case report, Schick et al, which was published from our institution, was added to the data. Agreement between the two investigators was 0.944 using Cohen's kappa coefficient and disagreements were resolved with discussion involving both investigators. A total of 33 patients with DGPs with lymph node metastases were identified (Figure 1).

\section{Patient demographics and characteristics}

The age of patients ranged from 16-74 years with a mean of 48 years and standard deviation of 15.3 years in 33 patients identified. Male to female ratio was 1:1. Of the cases that reported presenting symptoms, abdominal pain was the most common (57\%), followed by gastrointestinal bleeding/anemia (43\%), weight loss (13\%), obstructive jaundice (10\%), pancreatitis (7\%), duodenal obstruction (7\%), and incidental (7\%). Past medical history reported two patients with alcohol abuse, two patients with tobacco use, and two cases having the genetic traits of thalassemia and familial spastic paresis [12-15] (Table 1). 


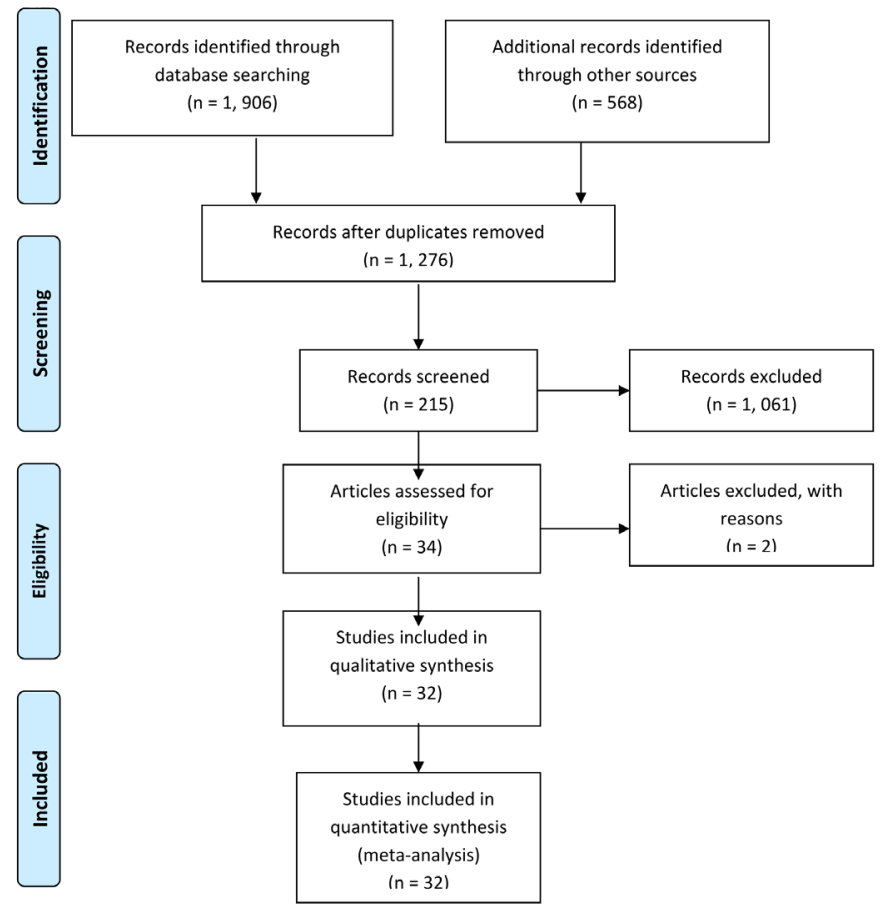

Figure 1: PRISMA 2009 flow Diagram.

The primary tumor was located in the second portion of the duodenum in $28 / 31$ cases ( $90 \%$ ), with 21 of reported DGPs being a ampullary/sub-ampullary/periampullary mass. Other described tumor locations were the third portion of duodenum, and two cases involving the head of the pancreas [16]. Reported primary tumor descriptions, obtained from endoscopy and pathology specimens indicated that $83 \%$ had mucosal ulceration and $95 \%$ had a polyploid or exophytic appearance. Mean tumor size (max diameter) was $3.1 \mathrm{~cm}$, SD 1.6, with sizes ranging from 1.0-9.0 cm (Table 1).

\section{Serum and urine tumor markers}

Serum/urine tumor markers and/or hormone levels were reported in six cases, including somatostatin, gastrin, pancreatic polypeptide, calcitonin, serotonin, dopamine, adrenaline, noradrenaline, chromogranin, CEA, Ca19-9, urine 5-HIAA, urinary metanephrines/ normetanephrines, and urine vanillylmandelic acid. These values were unremarkable, other than one case of elevated somatostatin, which normalized postoperatively [17]. As well, one case of elevated pancreatic polypeptide postoperatively, was thought to be indicative of tumor recurrence [18] (Table 2).

\section{Diagnostic imaging and biopsies}

Esophagogastroduodenoscopy was reported in 23 patients and successfully identified a polyploid duodenal mass in 23/23 cases (100\%). However, on 9 reported esophagogastroduodenoscopy biopsies, 1 suggested paraganglioma (11\%). Computed tomography scan was reported in 19 cases, detecting the tumor in 16/19 cases (84\%), and detecting enlarged lymph node in 8/17 cases
Table 1: Patient demographics, presenting complaint and tumor characteristics

\begin{tabular}{ll}
$\begin{array}{l}\text { Duodenal gangliocytic } \\
\text { paragangliomas with lymph node } \\
\text { metastases (n) }\end{array}$ & $\mathbf{3 3}$ patients \\
\hline Age (mean, SD, (range)) years & $\begin{array}{l}48,15 \cdot 3, \text { (16-74 } \\
\text { years) }\end{array}$ \\
Sex (n, Male/Female) & $32(16 / 16)$ \\
Presenting Complaint (n) & 30 \\
Abdominal Discomfort, n (\%) & $17(57 \%)$ \\
Gastrointestinal Bleeding/Anemia, n (\%) & $13(43 \%)$ \\
Weight Loss, n (\%) & $4(13 \%)$ \\
Obstructive Jaundice, n (\%) & $3(10 \%)$ \\
Duodenal Obstruction, n (\%) & $2(7 \%)$ \\
Pancreatitis, n (\%) & $2(7 \%)$ \\
Incidental Finding, n (\%) & $2(7 \%)$ \\
Size (max diameter cm) (mean, SD) & $3.1,1.6(1.0-9.0)$ \\
Triphasic cellular distribution, n (\%) & $33(100 \%)$ \\
Location & 31 \\
2nd portion of duodenum, n (\%) & $28,(96 \%)$ \\
Periampullary/Ampullary (n) & 21 \\
Mucosal ulceration, n (\%) & $19(83 \%)$ \\
Polyploid/Exophytic, n (\%) & $19(95 \%)$ \\
Distant metastases, n (\%) & $6(18 \%)$ \\
\hline
\end{tabular}

Table 2: Recorded serum and urine tumor markers

\begin{tabular}{|c|c|c|}
\hline Authors & Preoperative & Postoperative \\
\hline Buchler & Not recorded & $\begin{array}{l}\text { Serotonin }(-), \text { 5HIAA } \\
(-), \text { Gastrin }(-)\end{array}$ \\
\hline Ogata & $\begin{array}{l}\text { Serum markers are } \\
\text { within normal limits }\end{array}$ & Not recorded \\
\hline Tomic & $\begin{array}{l}\text { Somatostatin }(+++) \\
\text { Calcitonin, Vasoactive } \\
\text { intestinal peptide, } \\
\text { Pancreatic polypeptide, } \\
\text { Gastrin, Serotonin level } \\
\text { within normal limits }\end{array}$ & $\begin{array}{l}\text { Somatostatin } \\
\text { normalized }\end{array}$ \\
\hline Uchida & $\begin{array}{l}\text { Dopamine, adrenaline/ } \\
\text { noradrenaline, urine } \\
\text { catecholamines, CEA, } \\
\text { CA19-9 within normal } \\
\text { limits }\end{array}$ & Not recorded \\
\hline Amin & $\begin{array}{l}\text { Somatostatin and } \\
\text { chromogranin A are } \\
\text { within normal limits, } \\
\text { pancreatic polypeptide } \\
\text { not recorded }\end{array}$ & $\begin{array}{l}\text { Pancreatic polypeptide } \\
(+++) \text {, Gastrin, } \\
\text { Insulin, Glucagon, } \\
\text { Vasoactive intestinal } \\
\text { peptide, 5-HIAA, } \\
\text { Catecholamine, CG } \\
\text { within normal limits; }\end{array}$ \\
\hline Saito & $\begin{array}{l}\text { Glucagon, Gastrin, } \\
\text { Urinary 5-HIAA, A/ } \\
\text { NoA; Met/NoMet; } \\
\text { vanillylmandelic acid } \\
\text { within normal limits }\end{array}$ & Not recorded \\
\hline
\end{tabular}


(47\%). Endoscopic ultrasound was reported in 11 cases, and identified the primary tumor in $13 / 13$ cases (100\%), detecting enlarged lymph node in $7 / 11$ cases (64\%) (Table 3). Of nine biopsies reported by endoscopic ultrasound, gangliocytic paraganglioma was correctly diagnosed in one case, but suspicious/indeterminate in seven cases, and misdiagnosed as adenocarcinoma in another (Table 4). Other imaging included gastrografin swallow, MRI, Intraoperative ultrasound, PET scan, and Octreotide scan.

\section{Management and outcomes}

Local excision was performed in seven cases, however, three of these cases had a following pancreaticoduodenectomy, and one case had resection of the fourth portion of duodenum. Local excision required re-implantation of the pancreatic duct in two cases, and common bile duct in one case [19, 20]. Re-operation reasons included tumor recurrence, positive margins or surgeon/patient preference once lymph node involvement was discovered. Endoscopic excision was performed in two cases; both resulted in local failure and following pancreaticoduodenectomy. Pancreaticoduodenectomy was the definitive surgery in $27 / 31$ (87\%) of cases. The postoperative recovery was reported as unremarkable in $12 / 16$ (75\%) cases of pancreaticoduodenectomy, with complications reported in four cases, including, one mortality from cardiopulmonary failure (Table 3). Sites of metastases in 6 cases included liver, mesentery, pelvis, retroperitoneal, and bone. Local tumor recurrence was reported in one case, after local excision, with another case likely, but not definitively identified $[8,20]$. Even with recurrence at five and nine years, the tumor had appeared to progress slowly and there were relatively minimal symptoms with good outcomes $[1,8]$. The mean and median time postoperatively with no evidence of disease was 34 and 24 months. The mortality rate for the initial hospital stay was 3.3\%. Accounting for length of follow-up, one-year mortality was $4.3 \%(n=23)$; three-year mortality was $15 \%(n=13)$; five-year mortality was $25 \%(n=8)$. There were 3 mortalities/33 cases recorded from postoperative complications, metastatic disease, and a mortality of unknown cause at 156 months $[13,16,21]$ (Table 5).

\section{DISCUSSION}

We collected as many publications, in all languages, of duodenal paragangliomas with lymph node metastasis and reported a total of 33 cases. We examined the clinical and surgical management, exploring significant factors associated with diagnosis, treatment, progression and metastatic nature of these cases.

\section{Clinical presentation}

Patient presentation was similar to those without lymph node metastasis, with abdominal discomfort being
Table 3: Biopsies

\begin{tabular}{ll} 
Endoscopic biopsy & $\begin{array}{l}\text { Endoscopic ultrasound } \\
\text { biopsy }\end{array}$ \\
\hline $1 / 9$ correct $d x$ & $1 / 7$ correct $d x ; 1 / 7$ incorrect $d x ;$ \\
& $2 / 7$ suspicious but not $d x ; 5 / 8$ \\
& inconclusive.
\end{tabular}

slightly more common, closely followed by gastrointestinal bleeding and anemia [10]. Biliary obstruction, gastrointestinal obstruction, pancreatitis, and weight loss were also more common than in 173 duodenal gangliocytic paragangliomas reported by Okubo et al., likely reflecting the later presentation, increased tumor size from a mean of 2.5-3.15 cm and more extensive disease. Despite this, the majority of our cases still followed a slow indolent course, with $7 \%$ still being incidental findings, and recurrence occurring in only two cases at five and nine years $[1,8]$ (Table 1 and Table 3).

\section{Diagnostic imaging and biopsies}

Computed tomography scan was the initial diagnostic imaging of choice, in both diagnosis of a duodenal mass and detection of lymph node involvement. It should likely be involved in every workup, as well as postoperative monitoring. The few cases that reported MRCP or MRI were not superior in detecting lymph node involvement, however, may be useful in cases of obstructive jaundice to rule out other pathology and assess encroachment of the tumor on the biliary tract or pancreatic duct [10, 22, 23]. An Octreotide scan demonstrated positive uptake in one of two cases reported $[11,24]$. If positive, this modality may be useful in detecting tumor recurrence.

Esophagogastroduodenoscopy is a sensitive diagnostic modality for detecting a polyploid/ulcerated lesion in the duodenum. However, due to the submucosal location of the tumor, it is not specific and has no role in diagnosing gangliocytic paragangliomas by tissue biopsy, but may rule in/out other pathologies.

Endoscopic ultrasound detected the primary tumor in all reported cases, and described lymph node involvement in $64 \%$ of the cases. Endoscopic ultrasound biopsies were more specific, correctly identifying gangliocytic paraganglioma in one case, but recorded as suspicious/ indeterminate for gangliocytic paraganglioma in others. This reflects the relative difficulty for pathologists to diagnose a rare triphasic cellular tumor with a fine needle aspiration, with one case incorrectly diagnosing adenocarcinoma [16].

A core tissue sample is more useful, but difficult to obtain given the location of these tumors. There are reports of intraoperative ultrasound being utilized to detect lymph node metastasis, and may possibly offer a method to determine local excision versus pancreaticoduodenectomy if the tumor is correctly identified preoperatively as gangliocytic paraganglioma [25]. 
Table 4: Diagnostic techniques to detect duodenal gangliocytic paraganglioma +/- lymph node involvement

\begin{tabular}{|c|c|c|c|}
\hline Authors & Endoscopy & $\begin{array}{c}\text { (CT scan) } \\
\text { (Lymph node) }\end{array}$ & $\begin{array}{l}\text { (Endoscopic ultrasound) } \\
\text { (Lymph node) }\end{array}$ \\
\hline Dookhan & + & $(+)(-)$ & Not Reported \\
\hline Buchler & + & Not Reported & Not Reported \\
\hline Korbi & + & $(-)(-)$ & Not Reported \\
\hline Burke & Not Reported & Not Reported & Not Reported \\
\hline Burke & Not Reported & Not Reported & Not Reported \\
\hline Inai & Not Reported & Not Reported & Not Reported \\
\hline Hashimoto & + & Not Reported & Not Reported \\
\hline Takabayashi & + & Not Reported & Not Reported \\
\hline Tomic & Not Reported & $(+)(-)$ & Not Reported \\
\hline Sundararajan & + & $(+)(-)$ & Not Reported \\
\hline Henry & + & $(+)($ Not Reported) & Not Reported \\
\hline Bucher & + & Not Reported & Not Reported \\
\hline Wong & + & $(-)(-)$ & Not Reported \\
\hline Witkiewicz & + & Not Reported & Not Reported \\
\hline Mann & + & $(+)(+)$ & Not Reported \\
\hline Saito & + & $(+)(+)$ & $(+)(+)$ \\
\hline Uchida & + & $(+)(+)$ & $(+)($ Not Reported) \\
\hline Okubo & + & Not Reported & $(+)(+)$ \\
\hline Fiscaletti & Not Reported & $(+)(-)$ & $(+)(-)$ \\
\hline Rowsell & Not Reported & $(+)($ Not Reported) & Not Reported \\
\hline Ogata & Not Reported & Not Reported & $(+)(-)$ \\
\hline Barret & + & $(+)(+)$ & $(+)(+)$ \\
\hline Dustin & + & $(-)(-)$ & $(+)(-)$ \\
\hline Amin & Not Reported & $(+)(+)$ & $(+)(+)$ \\
\hline Park & Not Reported & Not Reported & Not Reported \\
\hline $\mathrm{Li}$ & + & $(+)(-)$ & Not Reported \\
\hline Shi & + & $(+)(+)$ & $(+)(+)$ \\
\hline Choi & + & Not Reported & Not Reported \\
\hline Wang & + & $(+)(+)$ & $(+)(-)$ \\
\hline Dowden & Not Reported & $(+)(-)$ & $(+)(+)$ \\
\hline Sun & + & Not Reported & $(+)($ Not Reported) \\
\hline $\mathrm{Hu}$ & + & Not Reported & Not Reported \\
\hline Schick & + & $(+)(+)$ & $(+)(+)$ \\
\hline
\end{tabular}

(+) diagnosis was made on imaging;

(-) diagnosis was not made on imaging.

\section{Serum and urine tumor markers}

Carcinoembryonic antigen and CA19-9 had no significant rise, and their role is limited to assisting in ruling out other malignancies. Of all serum and urine hormones measured, only somatostatin was confirmed to be elevated preoperatively, and dropped appropriately postoperatively in one case [17]. Pancreatic polypeptide levels increased with an increasing metastatic lesion to liver however more information is needed to determine the significance of pancreatic polypeptide $[18,26]$.
However, even with few reports of functioning tumors, the epithelioid component often contained neuroendocrine granules, which appear secretory [2, 3, 24]. With such little data available, it is reasonable to perform serum hormone levels, and if there are changes, monitor them postoperatively (Table 2).

As these tumors have functional qualities, it may be appropriate to follow with serum tumor markers if positive preoperatively, such as somatostatin and pancreatic polypeptide [18]. Octreotide scan if positive, 
Table 5: Operations and Outcomes

\begin{tabular}{lc} 
Local resection & 7 (23\%) \\
Endoscopic & 2 \\
Local resection with lymph node & 5 \\
dissection & \\
Need for 2nd operation & $4(57 \%)$ \\
Pancreatoduodenectomy & $27(87 \%)$ \\
Need for 2nd operation & $0(0 \%)$ \\
Perioperative mortality & $1 / 33$ \\
Outcomes (mortality) & 30 \\
1 year & $1 / 23(4.3 \%)$ \\
3 year & $2 / 13(15 \%)$ \\
5 year & $2 / 8(25 \%)$ \\
\hline
\end{tabular}

may also have a role in tumor surveillance. More evidence is needed to confirm the utility of these modalities in diagnosis and surveillance.

\section{Surgical treatment}

The current treatment of the majority of duodenal gangliocytic paragangliomas with lymph node metastases is pancreaticoduodenectomy with lymph node dissection. This is likely the most effective method of ensuring complete excision of the primary tumor and removal of affected lymph node.

Our review of these cases with lymph node metastases, still indicates that the majority of these tumors behave in a benign fashion with very slow progression, low recurrence rates and good outcomes. While DGPs may behave more aggressively, a pancreaticoduodenectomy is a high-risk operation with a reported mortality of $3-4 \%$ and morbidity rates of 30-60\%.

A discussion should occur with patients, considering co-morbidities, risks/benefits, and patient preference, as local excision +/- lymph node dissection may be a reasonable option in those who will not tolerate a pancreaticoduodenectomy procedure. If the tumor directly involves the ampulla, local excision is difficult as it may require re-implantation of the common bile duct and pancreatic duct. There are reports of endoscopic excision. However, with many reports indicating gangliocytic paragangliomas invade the muscularis propria and subserosa, the role of endoscopic surgery has yet to be established [1, 27-28]. Tumor location, depth of invasion, risks of procedure related complications and possible lymph node status should be considered [28]. Both cases of endoscopic excision in our review progressed to a pancreaticoduodenectomy for positive margins $[1,12]$. Distant metastatic sites included liver, mesentery, pelvis, retroperitoneal, and bone. They should be excised when possible, and recurrence is variable, with only one report of mortality, in which both chemotherapy and radiation were used, with minimal response [21]. The role of chemotherapy and radiation therapy is unknown, and decisions in this regard should be made after an informed discussion between patients and oncologists, including the uncertainty of these adjuncts [21, 23, 29].

\section{Histopathology and immunohistochemistry}

On histopathology, identification of epithelioid, spindle and ganglion cells were present in all primary tumors, which is a requirement for diagnosis of a gangliocytic paraganglioma. The epithelioid cell component predominates in the primary tumor, as well as metastases. These cells stain positive for neuron specific enolase, chromogranin A and synaptophysin. Spindle-shaped cells are positive for S-100. ganglion like cells uptake neuron specific enolase and synaptophysin and may stain positive for pancreatic polypeptide or somatostatin.

There are no confirmed histopathological or immunohistochemistry features at this time that predict lymph node metastases or more aggressive behavior. However, tumor invading beyond the muscularis propria, increased tumor size, and angiolymphatic invasion should increase the examiner's suspicion for more extensive disease.

\section{Origin}

Despite extensive immunohistochemistry and ultrastructural studies, the histogenesis of gangliocytic paragangliomas is still debatable, and authors have been unable to reconcile the combination of endocrine, ganglion and spindle cells observed in a single tumor [21]. The tumor components are of different embryologic origins, the first being of endodermal origin and the others originating from neural crest tissue. All components normally occur in the duodenum, and given the relatively benign nature, some contend that it is a hamartoma [30]. Others postulate it is a hyperplastic or neoplastic lesion from ectopic cells of the ventral primordium of the pancreas, possibly explaining its relatively consistent location in second portion of the duodenum [2, 9, 22]. Also suggested is an ectodermal origin, originating from pluripotent stem cells of the crypts of Lieberkuhn or celiac ganglion [1, 3, 5-7]. Gangliocytic paragangliomas discovered distant to the duodenum and pancreas, such as lung, esophagus, appendix, and spinal cord and the presence of metastatic lesions argue against a true hamartoma [30, 31]. Often regarded as a neuroendocrine tumor, it has rarely been shown to be a functional tumor, and neuroendocrine tumors lack ganglion and spindle cells [24]. While the epithelioid component is often immunopositive for pancreatic polypeptide, it is not specific to pancreatic neuroendocrine tumors [19].

\section{Outcomes}

It has been suggested that gangliocytic paraganglioma be defined as a relatively benign tumor with uncertain malignant potential. In our cases, there was one report of mortality, thought to be directly secondary to the 
tumor, one report from cardiopulmonary failure after pancreaticoduodenectomy procedure, and one report after 13 years of unknown cause $[13,16,21]$. We report relatively good one-year and five-year mortality rates, although we are limited by lack of extended outcomes. Only two cases showed evidence of local recurrence and 5/30 cases showed evidence of distance metastasis. This continues to suggest that gangliocytic paraganglioma, even with evidence of local spread with lymph node metastases, typically behave in a benign fashion. There is insufficient data to provide recommendations on timing of postoperative surveillance, however, those with more extensive disease should have their clinical status monitored closely, and be followed by diagnostic imaging to monitor for recurrence.

\section{LIMITATIONS}

There are limitations to our review. Diagnostic modalities, patient and tumor characteristics published may be biased, as there is a tendency to only publish positive results, therefore sensitivity, specificity and predictive values of tests were not calculated. With the majority of DGPs being treated with local excision, which does not allow examination of the node, the number of cases with lymph node metastasis may be underestimated. With only case reports in the medical literature, all recommendations were grade $\mathrm{C}$ [1].

\section{CONCLUSION}

Given there is no strong evidence in our review that distant metastases or lymph node involvement have additional clinicopathological indicators to suggest more aggressive behavior, it is difficult to predict malignant behavior of DGPs. Computed tomography scan and endoscopic ultrasound have a role in detecting a mass and aid in detecting the lymph node involvement. Preoperative tissue diagnosis is difficult, and endoscopic ultrasound fine needle aspirates are often inconclusive. Endoscopy is sensitive for detecting a lesion, but is diagnostically limited by the submucosal location of the tumor. Extended outcomes have been shown to be quite good in our review, and the presence of lymph node metastases does not seem to influence the prognosis, but rather the treatment modality. In the presence of duodenal gangliocytic paragangliomas with lymph node metastases, pancreaticoduodenectomy is likely the most effective method of ensuring complete tumor excision and removal of affected lymph node. Local excision with lymph node dissection may be effective in those who will not tolerate a pancreaticoduodenectomy. An informed discussion of the limited evidence of pancreaticoduodenectomy versus local excision should occur, with patient comorbidities, risks/benefits and patient preference being taken into consideration. Treating clinicians should be aware of the uncertain malignant potential, and postoperatively closer monitoring of the patient is recommended.

\section{REFERENCES}

1. Sundararajan V, Robinson-Smith TM, Lowy AM. Duodenal gangliocytic paraganglioma with lymph node metastasis: A case report and review of the literature. Arch Pathol Lab Med 2003 Mar;127(3):e139-41.

2. Perrone T, Sibley RK, Rosai J. Duodenal gangliocytic paraganglioma: An immunohistochemical and ultrastructural study and a hypothesis concerning its origin. Am J Surg Pathol 1985 Jan;9(1):31-41.

3. Scheithauer BW, Nora FE, LeChago J, et al. Duodenal gangliocytic paraganglioma: Clinicopathologic and immunocytochemical study of 11 cases. Am J Clin Pathol 1986 Nov;86(5):559-65.

4. Sánchez-Pérez MA, Luque-de León E, MuñozJuárez M, Moreno-Paquentin E, Genovés-Gómez H, Torreblanca-Marín MA. Duodenal gangliocytic paraganglioma. Can J Surg 2009 Apr;52(2):E27-8.

5. Lukash WM, Hyams VJ, Nielsen OF. Neurogenic neoplasms of the small bowel: Benign nonchromaffin paraganglioma of the duodenum. Am J Dig Dis 1966 Jul;11(7):575-9.

6. Morita T, Tamura S, Yokoyama Y, et al. Endoscopic resection of a duodenal gangliocytic paraganglioma. Dig Dis Sci 2007 Jun;52(6):1400-4.

7. Nakamura T, Ozawa T, Kitagawa M, et al. Endoscopic resection of gangliocytic paraganglioma of the minor duodenal papilla: Case report and review. Gastrointest Endosc 2002 Feb;55(2):270-3.

8. Dookhan DB, Miettinen M, Finkel G, Gibas Z. Recurrent duodenal gangliocytic paraganglioma with lymph node metastases. Histopathology 1993 Apr;22(4):399-401.

9. Shi H, Han J, Liu N, et al. A gangliocytic patially glandular paraganglioma with lymph node metastasis. Diagn Pathol 2014 Mar 20;9:63.

10. Okubo Y, Wakayama M, Nemoto T, et al. Literature survey on epidemiology and pathology of gangliocytic paraganglioma. BMC Cancer 2011 May 20;11:187.

11. Phillips B, Ball C, Badenoch D, Straus S, Haynes B, Dawes M. Oxford centre for evidence-based medicine levels of evidence. BJU Int 2001;107(5):870.

12. Witkiewicz A, Galler A, Yeo CJ, Gross SD. Gangliocytic paraganglioma: Case report and review of the literature. J Gastrointest Surg 2007 Oct;11(10):13514 .

13. Korbi S, Kapanci Y, Widgren S. Malignant paraganglioma of the duodenum: Immunohistochemical and ultrastructural study of a case. [Article in French]. Ann Pathol 1987;7(1):47-55.

14. Fiscaletti M, Fornelli A, Zanini N, et al. Segmental groove pancreatitis and duodenal gangliocytic paraganglioma with lymph node metastasis: A newly described association. Pancreas 2011 Oct;40(7):11457.

15. Saito J, Hirata N, Furuzono M, et al. A case of duodenal gangliocytic paraganglioma with lymph node metastasis. [Article in Japanese]. Nihon Shokakibyo Gakkai Zasshi 2010 Apr;107(4):639-48. 
16. Henry C, Ghalel-Méchaoui H, Bottero N, Pradier $\mathrm{T}$, Moindrot H. Gangliocytic paraganglioma of the pancreas with bone metastasis. Ann Chir 2003 Jun;128(5):336-8.

17. Tomic S, Warner T. Pancreatic somatostatin-secreting gangliocytic paraganglioma with lymph node metastases. Am J Gastroenterol 1996 Mar;91(3):6078.

18. Amin SM, Albrechtsen NW, Forster J, Damjanov I. Gangliocytic paraganglioma of duodenum metastatic to lymph nodes and liver and extending into the retropancreatic space. Pathologica 2013 Jun;105(3):90-3.

19. Inai K, Kobuke T, Yonehara S, Tokuoka S. Duodenal gangliocytic paraganglioma with lymph node metastasis in a 17-year-old boy. Cancer 1989 Jun $15 ; 63(12): 2540-5$.

20. Büchler M, Malfertheiner P, Baczako K, Krautzberger W, Beger HG. A metastatic endocrine-neurogenic tumor of the ampulla of Vater with multiple endocrine immunoreaction: Malignant paraganglioma? Digestion 1985;31(1):54-9.

21. Li B, Li Y, Tian XY, Luo BN, Li Z. Malignant gangliocytic paraganglioma of the duodenum with distant metastases and a lethal course. World $\mathrm{J}$ Gastroenterol 2014 Nov 7;20(41):15454-61.

22. Bucher P, MatheZ, Bühler L, et al. Paraganglioma of the ampulla of Vater: A potentially malignant neoplasm. Scand J Gastroenterol 2004 Mar;39(3):291-5.

23. Wong A, Miller AR, Metter J, Thomas CR Jr. Locally advanced duodenal gangliocytic paraganglioma treated with adjuvant radiation therapy: Case report and review of the literature. World J Surg Oncol 2005 Mar 1;3(1):15.

24. Dustin SM, Atkins KA, Shami VM, Adams RB, Stelow EB. The cytologic diagnosis of gangliocytic paraganglioma: A case report. Diagn Cytopathol 2013 Jul;41(7):650-3.

25. Hashimoto S, Kawasaki S, Matsuzawa K, Harada $\mathrm{H}$, Makuuchi M. Gangliocytic paraganglioma of the papilla of Vater with regional lymph node metastasis. Am J Gastroenterol 1992 Sep;87(9):1216-8.

26. Ljungberg O, Järnerot G, Rolny P, Wickbom G. Human pancreatic polypeptide (HPP) immunoreactivity in an infiltrating endocrine tumour of the papilla of Vater with unusual morphology. Virchows Arch A Pathol Anat Histol 1981;392(1):119-26.

27. Hu W, Gao S, Chen D, et al. Duodenal gangliocytic paraganglioma with lymph node metastases: A case report and review of literature. Int J Clin Exp Pathol 2016;9(4):4756-60.

28. Wang B, Zou Y, Zhang H, Xu L, Jiang X, Sun K. Duodenal gangliocytic paraganglioma: Report of two cases and review of literature. Int J Clin Exp Pathol 2015 Sep 1;8(9):9752-9.
29. Rowsell C, Coburn N, Chetty R. Gangliocytic paraganglioma: A rare case with metastases of all 3 elements to liver and lymph nodes. Ann Diagn Pathol 2011 Dec;15(6):467-71.

30. Hironaka M, Fukayama M, Takayashiki N, Saito K, Sohara Y, Funata N. Pulmonary gangliocytic paraganglioma: Case report and comparative immunohistochemical study of related neuroendocrine neoplasms. Am J Surg Pathol 2001 May;25(5):688-93.

31. Takabayashi N, Kimura T, Yoshida M, Sakuramachi S, Harada Y, Kino I. A case report of duodenal gangliocytic paraganglioma with lymph node metastasis. Jpn Soc Gastroenterol Surg 1993;26:2444-8.

$* * * * * * * * *$

\section{Author Contributions}

Luke Hartford - Substantial contributions to conception and design, Acquisition of data, Analysis and interpretation of data, Drafting the article, Final approval of the version to be published

Alexsi Sherazadishvili - Substantial contributions to conception and design, Acquisition of data, Analysis and interpretation of data, Drafting the article, Final approval of the version to be published

Ken Leslie - Substantial contributions to conception and design, Acquisition of data, Analysis and interpretation of data, Drafting the article, Final approval of the version to be published

\section{Guarantor of Submission}

The corresponding author is the guarantor of submission.

\section{Source of Support}

None

\section{Conflict of Interest}

Authors declare no conflict of interest.

\section{Copyright}

(C) 2017 Luke Hartford et al. This article is distributed under the terms of Creative Commons Attribution License which permits unrestricted use, distribution and reproduction in any medium provided the original author(s) and original publisher are properly credited. Please see the copyright policy on the journal website for more information. 
Access full text article on other devices

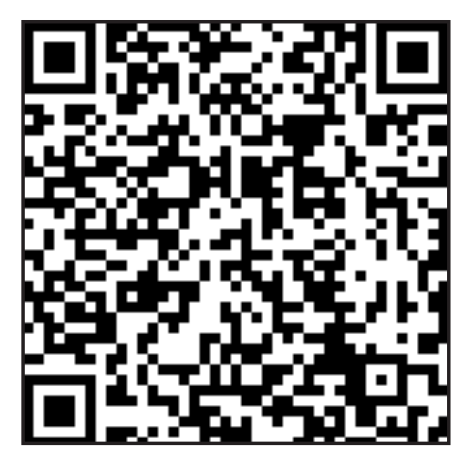

Access PDF of article on other devices

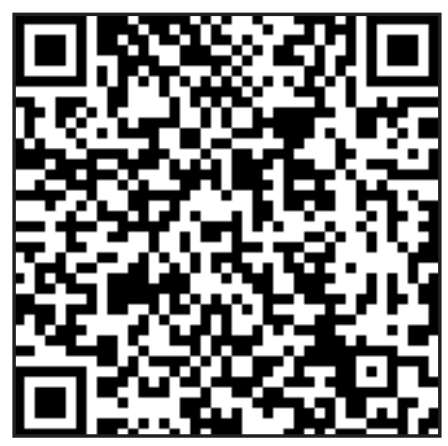

